\title{
Stroke Following Radiofrequency Ablation of the Great Saphenous Vein
}

\author{
Seth Noland MD*, Charles Hartranft DO and Michael Kunstmann DO
}

Department of Surgery, Mount Carmel Health System, Columbus, Ohio, USA

\begin{abstract}
A 55-year-old woman underwent radiofrequency ablation of her great saphenous vein and phlebectomy. Upon recovery from anesthesia, the patient was unable to speak but could follow commands. Immediate magnetic resonance imaging showed small diffusion abnormalities in the left frontal lobe and parietal lobe cortex consistent with acute left-sided infarcts. Further evaluation showed normal carotid velocities with no evidence of stenosis and no plaque. Duplex scan showed no deep vein thrombus (DVT) in either extremity. However, transesophageal echocardiogram showed a patent foramen ovale. The patient was started on anticoagulation medication and had recovered to baseline status by hospital day six, allowing for discharge.
\end{abstract}

Keywords: Stroke; Radiofrequency ablation; Patent foramen ovale

\section{Introduction}

Chronic venous disease is an important pathologic condition seen routinely by vascular surgeons. Varicose veins in the great saphenous vein (GSV) distribution are the most common manifestations of chronic venous disease and are present in $20 \%$ to $25 \%$ of women and in $10 \%$ to $15 \%$ of men [1]. Saphenous reflux was once treated routinely with saphenofemoral ligation and vein stripping but now can be managed with multiple less-invasive therapies. Endovenous laser therapy (EVLT), radiofrequency ablation (RFA), and foam sclerotherapy are just as effective as the open procedure with less morbidity, faster recovery, and improved cosmetic results [2]. Minimally invasive treatments aim to occlude the saphenous vein; however, high ligation of the great saphenous vein is not usually performed with these procedures. Radiofrequency ablation and EVLT offer comparable venous occlusion rates at three months after treatment of primary GSV varices with no statistical difference in efficacy. Radiofrequency ablation is associated with less periprocedural pain, analgesic requirement and bruising, making it the treatment of choice at some institutions [3]. The RFA procedure is associated with the perioperative risk of paraesthesia ( $4 \%$ to $20 \%$ ), phlebitis ( $7 \%$ to $9 \%$ ), and bruising or skin pigmentation changes $(6 \%$ to $19 \%)$. In addition, there is risk of endovenous heatinduced thrombosis and deep vein thrombosis (DVT) with a previously reported incidence of $0.3 \%$ after EVLT and $2.1 \%$ after RFA [4,5]. This report presents a patient with an unknown patent foramen ovale (PFO) who underwent RFA of the great saphenous vein complicated by leftsided cerebral infarcts.

\section{Case Report}

A 55-year-old woman was evaluated in the office for right lower extremity discomfort associated with noticeable varicose veins. A lower extremity venous duplex scan did not display any evidence of DVT but did identify incompetence of the great saphenous vein with a reflux time of 5975 milliseconds. The patient was advised to wear sequential compression stockings in an attempt to achieve symptomatic improvement. A follow-up visit found no improvement and the patient was scheduled for RFA of her GSV as well as phlebectomy of her varicose veins.

The RFA procedure utilized the VNUS ClosureFAST ${ }^{\mathrm{TM}}$ Radiofrequency Catheter (Covidien, Mansfield, MA, USA) and the phlebectomy utilized the TRIVEX ${ }^{\mathrm{TM}}$ System (InaVein, Lexington, MA,
USA). The position of the catheter measured $2.5 \mathrm{~cm}$ from the deep system. Ultrasound at the completion of the procedure showed no DVT but did identify clot within the great saphenous vein. There were no issues intraoperatively; however, the patient was slow to recover from anesthesia. Upon awakening, the patient was aphasic but could follow commands. An immediate computed tomographic (CT) brain scan displayed no abnormalities. Magnetic resonance imaging showed small diffusion abnormalities in the periphery of the left frontal and parietal lobe cortex consistent with acute left-sided infarcts (Figure 1).

Further evaluation for the source of embolism included carotid artery duplex, lower extremity venous duplex (Figure 2), transthoracic echocardiogram (TTE), and transesophageal echocardiogram (TEE). The carotid arteries had normal velocities with no evidence of stenosis and no plaque identified on B-mode imaging. The lower extremity venous duplex scan showed no DVT in either extremity; in particular, there was no DVT in the common femoral vein on the side in which RFA was performed. There was no evidence of atrial or ventricular thrombus on the TTE, but there did appear to be a PFO during the

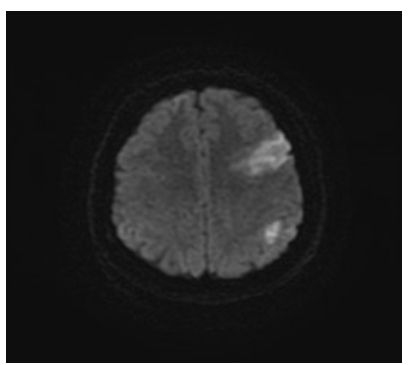

Figure 1: MRI displaying infarct in left frontal and parietal regions.

*Corresponding author: Seth Noland MD, Department of Surgery, Mount Carmel Medical Center, 793 West State St, Columbus, OH 43222, USA, Tel: 614-8890189; E-mail: snoland6@gmail.com

Received March 18, 2014; Accepted April 26, 2014; Published April 28, 2014

Citation: Seth Noland MD, Charles Hartranft DO, Michael Kunstmann DO (2014) Stroke Following Radiofrequency Ablation of the Great Saphenous Vein. J Vasc Med Surg 2: 136. doi: 10.4172/2329-6925.1000136

Copyright: (C) 2014 Seth Noland MD, et al. This is an open-access article distributed under the terms of the Creative Commons Attribution License, which permits unrestricted use, distribution, and reproduction in any medium, provided the original author and source are credited. 


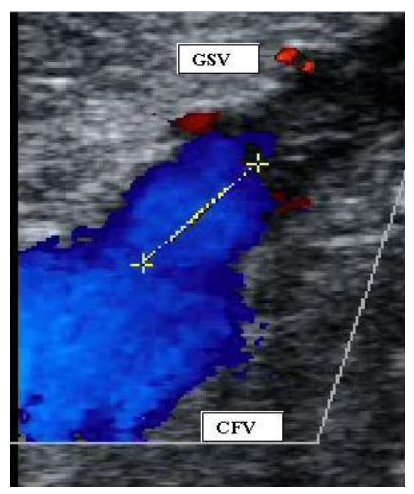

Figure 2: Postoperative duplex demonstrating patency of saphenofemoral junction with $1.1 \mathrm{~cm}$ distance from thrombus to junction.

bubble study, which was confirmed by a follow-up TEE with bubble study.

The patient improved daily, regaining ability to speak short sentences by postoperative day three and returning nearly to baseline by postoperative day six. A CT of the brain on postoperative day six found the brain to be stable with no new areas of ischemia. The patient was discharged on postoperative day six receiving oral anticoagulation therapy. She is now nine months post-RFA with no residual deficits or additional events.

\section{Discussion}

Although EVLT, RFA, and foam sclerotherapy are just as effective as the open procedure, they are not without risk of complication, as described. These risks range from ecchymosis to DVT and thromboembolism. The risk of DVT after RFA has been reported to be as low as $0 \%$ in some studies, to as high as $16 \%$ (12 of 73 patients) in a study by Hingorani et al. [4-6]. Jacobs et al. [7] performed a retrospective analysis of patients undergoing RFA procedures using the VNUS system. On postoperative ultrasound, thrombus protrusion into the deep system was found in 11 patients (4\%) and complete occlusion of the femoral vein noted in 2 patients $(0.7 \%)$. The only risk factor associated with postprocedural DVT was a previous history of DVT [7]. The exact incidence of DVT is difficult to determine, but most clinics quote risks of approximately 1:1000 and provide perioperative prophylaxis [4]. The fact that high ligation of the great saphenous vein is not routinely performed with any minimally invasive endovenous procedure can be seen as a potential cause of thromboembolic events.

Stroke following endovenous therapy has been reported previously in the literature, specifically with ultrasound-guided sclerotherapy (UGS). Ma et al. [8] describe a series of three patients experiencing right middle cerebral artery strokes following UGS with tetradecyl sulfate foam. All patients were found to have a PFO and all recovered completely [8]. Regan et al. performed a prospective study using microfoam ablation with periprocedural transcranial doppler and postoperative MRI to determine the incidence and significance of gas emboli [9]. Of the 61 patients found to have a right-to-left cardiac shunt, $54(89 \%)$ were noted to have middle cerebral artery gas emboli and 6 of the $21(29 \%)$ patients without a shunt had emboli on doppler studies. None of the 60 patients developed neurologic symptoms and none of the patients were found to have lesions on postoperative MRI. The authors concluded that arterial gas emboli can not be avoided during injection of sclerosants but the characteristics of the ultra-low nitrogen microfoam appeared to be safe as no periprocedural neurologic events were noted [9].

A report of stroke following EVLT of varicose veins was published in 2010 by Caggiati and Franceshini [10]. The patient had undergone successful laser ablation with obliteration of the GSV and had an uncomplicated early postoperative course. On postoperative day five, she suddenly developed right-side hemiparesis with global aphasia and complete occlusion of the left middle cerebral artery at the origin. The patient underwent bilateral lower extremity venous duplex with no DVT identified and a carotid artery duplex, which did not identify any area of occlusion or stenosis. A TEE with bubble study displayed a PFO with right-to-left shunt. After one year, the patient had ongoing aphasia and severe hemiparesis [10].

The patient in the present report underwent RFA of the GSV in combination with transilluminated power phlebectomy. Complications following transilluminated power phlebectomy vary considerably and consist primarily of ecchymosis and/or hematoma formation $(4.9 \%$ to $95 \%)$, paresthesias or nerve injury (9.5\% to $39 \%)$, skin perforation (1.2\% to $5 \%)$, superficial phlebitis $(2.4 \%$ to $13 \%)$, swelling ( $5 \%$ to $17.5 \%)$, hyperpigmentation ( $1.2 \%$ to $3.3 \%)$, and DVT $(<1 \%)$ [11] Passman et al. [12] compared the overall complication rate between combined saphenous stripping-stab avulsion phlebectomy, strippingtransilluminated-powered phlebectomy, and endovenous ablationtransilluminated-powered phlebectomy. There were no significant differences in DVT risk with percentages of $1.4 \%, 1.1 \%$, and $0.8 \%$, respectively [12].

The prevalence of a PFO has been evaluated in two large autopsy studies [13]. The first study included 51,100 participants and grouped the PFOs by size. A probe patent PFO measuring $0.2 \mathrm{~cm}-0.5 \mathrm{~cm}$ was found in $29 \%$ and a pencil patent PFO measuring $0.6 \mathrm{~cm}-1.0 \mathrm{~cm}$ was identified in 6\%. The second study with 5965 subjects recorded the PFO incidence of $27.3 \%$ with sizes ranging from $1 \mathrm{~mm}-19 \mathrm{~mm}$ [13]. The prevalence of right-to-left shunt was evaluated in 221 participants with GSV incompetence and varicose veins by Wright et al. [14]. Eightyfive (38.5\%) patients had a right-to-left shunt at rest and $114(51.8 \%)$ were positive after Valsalva maneuver. A total of 130 patients $(58.8 \%)$ had a right-to-left shunt, a much higher prevalence than in the general population [14].

Ischemic stroke following RFA of the great saphenous vein has not been reported previously in the literature. In the present case, a patient with no history of PFO was found to be aphasic in the post-anesthesia care unit and was diagnosed with multiple areas of small left-sided cerebral infarct. The hospital course included evaluation with a carotid duplex ultrasound, lower extremity venous duplex, TTE, and TEE with no clear source of embolus identified. However, it was believed that the most likely cause was thrombus originating from the ablation or gas emboli created during the procedure. The patient returned to baseline clinically by hospital day six and was discharged receiving oral anticoagulation therapy.

The case demonstrates a scenario in which a patient suffered a major complication from a minimally invasive procedure. The prevalence of PFO in the patient population with concomitant GSV reflux is higher than anticipated. Currently, however, there does not appear to be strong evidence to support routine preoperative evaluation with TEE and bubble study due to the high cost and low overall complication rate of venous thromboembolism. 
Citation: Seth Noland MD, Charles Hartranft DO, Michael Kunstmann DO (2014) Stroke Following Radiofrequency Ablation of the Great Saphenous Vein. J Vasc Med Surg 2: 136. doi: 10.4172/2329-6925.1000136

\section{Acknowledgement}

This research received no specific grant from any funding agency in the public, commercial, or not-for-profit sectors.

\section{References}

1. Callam MJ (1994) Epidemiology of varicose veins. Br J Surg 81: 167-173.

2. Brown K, Moore CJ (2009) Update on the treatment of saphenous reflux: laser, RFA or foam? Perspect Vasc Surg Endovasc Ther 4: 226-231.

3. Nordon IM, Hinchliffe RJ, Brar R, Moxey P, Black SA, et al. (2011) A prospective double-blind randomized controlled trial of radiofrequency versus laser treatment of the great saphenous vein in patients with varicose veins. Ann Surg 254: 876-881.

4. Anwar MA, Lane TR, Davies AH, Franklin IJ (2012) Complications of radiofrequency ablation of varicose veins. Phlebology 27 Suppl 1: 34-39.

5. Mozes G, Kalra M, Carmo M, Swenson L, Gloviczki P (2005) Extension of saphenous thrombus into the femoral vein: A potential complication of new endovenous ablation techniques. J Vasc Surg 41: 130-135.

6. Hingorani AP, Ascher E, Markevich N, Schutzer RW, Kallakuri S, et al. (2004) Deep venous thrombosis after radiofrequency ablation of greater saphenous vein: a word of caution. J Vasc Surg 40: 500-504
7. Jacobs CE, Pinzon MM, Orozco J, Hunt PJ, Rivera A, et al. (2014) Deep venous thrombosis after saphenous endovenous radiofrequency ablation: Is it predictable? Ann Vasc Surg 28: 679-685.

8. Ma RW, Pilotelle A, Paraskevas P, Parsi K (2011) Three cases of stroke following peripheral venous interventions. Phlebology 26: 280-284.

9. Regan JD, Gibson KD, Rush JE, Shortell CK, Hirsch SA, et al. (2011) Clinical significance of cerebrovascular gas emboli during polidocanol endovenous ultra-low nitrogen microfoam ablation and correlation with magnetic resonance imaging in patients with right-to-left shunt. J Vasc Surg 53: 131-138.

10. Caggiati A, Franceschini M (2010) Stroke following endovenous laser treatment of varicose veins. J Vasc Surg 51: 218-220.

11. Passman M (2007) Transilluminated powered phlebectomy in the treatment of varicose veins. Vascular 15: 262-268.

12. Passman MA, Dattilo JB, Guzman RJ, Naslund TC (2007) Combined endovenous ablation and transilluminated powered phlebectomy: is less invasive better? Vasc and Endovasc Surg 41: 41-47.

13. Kerut EK, Norfleet WT, Plotnick GD, Giles TD (2001) Patent foramen ovale: a review of associated conditions and the impact of physiological size. J Am Coll Cardiol 38: 613-623

14. Wright DD, Gibson KD, Barclay J, Razumovsky A, Rush J, et al. (2010) High prevalence of right-to-left shunt in patients with symptomatic great saphenous incompetence and varicose veins. J Vasc Surg 51: 104-107. 